\title{
Effect of Biarum carduchrum extract on brain tissue thiol level in rat model of 6-hydroxydopamine-induced Parkinson's disease
}

\author{
Zahra Zanganehnejad ${ }^{1}$, Mahbubeh Setorki ${ }^{*}$ \\ ${ }^{1}$ Department of Biology, Shahrekord Branch, Islamic Azad University, Shahrekord, Iran \\ ${ }^{2}$ Department of Biology, Izeh Branch, Islamic Azad University, Izeh, Iran
}

\section{A R T I C L E I N F O}

Article Type:

Original Article

Article History:

Received: 9 December 2017

Accepted: 13 May 2018

\section{Keywords:}

Biarum carduchrum

Medicinal plants

Parkinson's disease

Thiol

\begin{abstract}
A B S T R A C T
Introduction: Parkinson's disease (PD) is developed due to the degeneration of dopaminergic neurons in the substantia nigra, which is widely associated with oxidative stress. The reduction of glutathione, as the most abundant and important tissue thiol and an antioxidant defense agent, is one of the first biochemical events associated with PD, and therefore using antioxidants has a protective effect. In the present study, the effects of Biarum carduchrum extract on the thiol levels in the tissues of the hippocampus, striatum, cortex, and cerebellum in PD were investigated.

Methods: Wistar rats were randomly divided into 5 groups of 8 each. Control group was left without lesion. PD group received $8 \mu \mathrm{g}$ of neurotoxin-6-hydroxydopamine in the anteriormiddle-brain. Seven days after PD induction, groups 3-5 intragastrically received 100, 200 , and $400 \mathrm{mg} / \mathrm{mL}$ of B. carduchrum extract, respectively, for 14 days. At completion of treatments, brain tissues were removed and their thiol levels measured.

Results: The levels of thiol in cortex, cerebellum, hippocampus, and striatum were significantly lower in the rats with PD than the control group. In B. carduchrum extract-treated groups, the thiol levels of cerebral cortex, cerebellum, hippocampus, and striatum tissues increased significantly compared to PD group.

Conclusion: It seems that B. carduchrum extract leads to the functional regeneration of the nigro-striatal pathway through the mechanisms associated with its antioxidant properties.
\end{abstract}

Implication for health policy/practice/research/medical education:

Biarum carduchrum extract leads to functional regeneration of the nigro-striatal pathway probably through the mechanisms associated with its antioxidant properties. The results showed that B. carduchrum might be effective in Parkinson's disease.

Please cite this paper as: Zanganehnejad Z, Setorki M. Effect of Biarum carduchrum extract on brain tissue thiol level in rat model of 6-hydroxydopamine-induced Parkinson's disease. J Herbmed Pharmacol. 2018;7(3):136-140. doi: 10.15171/jhp.2018.23.

\section{Introduction}

Parkinson's disease (PD) is the most common neuropathological disorder that is developed due to the degeneration of dopaminergic neurons in the dense part of the substantia nigra and its terminals in the striatum (1). Reduction of dopamine leads to various motor and debilitating disorders such as bradykinesia, tremor, muscle rigidity, and the body's position imbalance (2). Currently, there is no definite treatment for neuronal cell death or PD. Currently available treatments only delay onset of disease or improve motor symptoms through increasing dopamine.

One of the family-related causes of $\mathrm{PD}$ is the mutation in the PARK2 gene, which encodes a protein called parkin. This protein is one of the components of the ubiquitin E3 ligase complex, which plays a role in the proteasome pathway. It is believed that oxidative stress is the cause of changes in this gene (3). The mechanism explained for the degeneration of dopaminergic neurons indicates that oxidative and nitrative stress can nitrosylate the MEF2C transcription factor, which inhibits the transcription of certain genes including PGC-1a. Inhibiting the expression of this protein causes a mitochondrial defect in the dopaminergic neurons and the cell to move toward apoptosis (3).

It is thought that free radicals are topically produced in

*Corresponding author: Mahbubeh Setorki,

Email: doctor.setorgi@gmail.com 
the basal nuclei, and free radical-induced oxidative stress leads to progressive damage and death of the dopaminergic neurons in the substantia nigra of PD patients. On the other hand, intrinsic defense mechanisms lead to increase in dopamine oxidation, neuromelanin production, and iron concentration, decrease in ferritin concentration and glutathione (GSH) production as well as increase in oxidized GSH. GSH depletion in the substantia nigra in $\mathrm{PD}$ is the result of neuronal degeneration. Different tissues including brain tissue have both an intracellular and extracellular thiol redox-regulating system that protects sulfhydryl (SH)-containing proteins from over-oxidation. For example, $\mathrm{SH}$ group donors and low-molecular-weight enzymes can catalyze the regeneration of $\mathrm{SH}$ groups in proteins and eliminate the toxicity of pro-oxidants, which are an important group of free radicals, by binding glutathione to the GSH regeneration. Intracellular GSH levels modulate cellular responses to oxidative stress and GSH depletion exacerbates oxidative stress (4).

Currently, vigorous attempts are being made for using medicinal plants to decrease the dose of dopaminergic drugs. Pharmacological research to date has only led to the synthesis of drugs that relieve the symptoms of PD while have little effect in preventing neuro-pathological changes. It is therefore necessary to seek out new therapeutic agents to reduce neuro-degeneration in the pathology of this disease (5).

Biarum carduchrum is a plant from family Areaceae that wildly occurs in the hillsides of Zagros mountains in the provinces of Fars, and Kohgiluyeh and Boyer-Ahmad, and is also distributed in some parts of Turkey, Syria, and Iraq (6). Williams et al were first to identify flavonoids and anthocyanins in the plants from family Areaceae (7). Besides that, these plants contain alkaloids and amines, saponin, cinnamic acids, and flavonoids. Flavonoids represent a group of compounds that are widely available in nature. The biological activities of flavonoids are related to their antioxidant properties.

Plants are valuable sources of phenolic compounds. These compounds protect the cells against oxidative stress through their antioxidant activities. Diets containing antioxidants are the best protection against oxidative stress and also protect the biological macromolecules (8). To the best of our knowledge, no study has yet been conducted on B. carduchrum effect on animal model of $\mathrm{PD}$, and therefore we were encouraged to investigate the effects of different doses of $B$. carduchrum extract on thiol levels in various regions of the brain in rat model of PD.

\section{Materials and method}

\section{Extraction of Biarum carduchrum}

Biarum carduchrum samples were collected from the vicinity of Izeh County and then botanically identified as B. carduchrum at Islamic Azad University, Izeh Branch, Izeh, Iran. Then, the leaves were separated and shadow dried in the open air. After the leaves were dried, a specific amount of them was weighed and finely pulverized into particles with a diameter of less than $0.4 \mathrm{~mm}$ by an electric mill. The resulting powder was macerated in $70 \%$ ethanol at room temperature for 72 hours. A mixture of alcohol and $B$. carduchrum powder was well stirred several times each day. Then, we passed the mixture through a filter to separate the extract. The resulting extract was distilled in vacuum so that its alcohol was completely evaporated. After alcohol was completely evaporated, the extract was obtained as a brown powder. The purity of the extract was calculated at $28 \%(9)$.

\section{Rats grouping}

Male Wistar rats weighing 200-250 g were housed at $21 \pm 2^{\circ} \mathrm{C}$ on a 12 -hour light: 12 -hour darkness cycle as they had free access to the same food and water. The rats were randomly assigned to five groups of eight each. Control group was left without lesion. PD group received $8 \mu \mathrm{g}$ of neurotoxin-6-hydroxydopamine in the anterior-middlebrain (MFB). Seven days after PD induction, the groups 3-5 intragastrically received 100,200 , and $400 \mathrm{mg} / \mathrm{mL}$ of B. carduchrum extract, respectively, for 14 days. The doses of the extract were selected with respect to a previous study (9).

\section{Inducing Parkinson's disease in rats}

The rats were anesthetized by intraperitoneal coadministration of $90 \mathrm{mg} / \mathrm{kg}$ of ketamine hydrochloride and $10 \mathrm{mg} / \mathrm{kg}$ of xylazine. They were then placed separately in a stereotaxic instrument and fixed by the mouthpiece and the bars inside the headphone and the hairs on the back of the skull shaved.

The scalp was first disinfected with alcohol and then a longitudinal incision made from the back surface of the head between the eyes to the back middle surface of the ears. The connective tissues on the skull surface were removed to make the Bergma point visible. The Bergma and Lambda points were placed on an equal surface at which the device marker was set. According to Atlas of Neurosurgical Techniques, the anterior-middle-brain (MFB) was determined at coordinates MFB (AP: 3/8, ML: $\pm 1 / 8$, DV:- $8 / 3$ ). PD was induced by unilaterally injecting $8 \mu \mathrm{g}$ of neurotoxin-6-hydroxydopamine in the MFB (10).

\section{Measuring brain thiol level}

The hippocampus, striatum, cortex, and cerebellum of each rat were removed and immediately weighed. One gram of each tissue was homogenized in $10 \mathrm{~mL}$ of KCL $1.5 \%$ solution. Of the homogenized solution, $0.5 \mathrm{~mL}$ was dissolved in $2.5 \mathrm{~mL}$ of TCA $3 \%$ and the resulting solution kept in a bain-marie at $37^{\circ} \mathrm{C}$ for 10 minutes and then centrifuged at $3000 \mathrm{rpm}$ for 10 minutes. The supernatant was isolated to measure thiol level using DTNB. In a test tube, $1 \mathrm{~mL}$ of Tris buffer was added to $50 \mu \mathrm{L}$ of the 
homogenized tissue solution and then optical absorbance was read at 412 -nm wavelength (A1). Then, $20 \mu \mathrm{L}$ of DTNB was poured in the tubes, the resulting solution allowed to stand at room temperature for 15 minutes, and its absorbance was read at 412-nm wavelength (A2). The absorbance of control was also read at 412-nm wavelength (B). Thiol levels were measured by the equation below: Thiol $=(\mathrm{A} 2-\mathrm{A} 1-\mathrm{B}) * 1.07 / 0.05^{*} 13.6$

\section{Statistical analysis}

Data analysis was conducted by one-way analysis of variance (ANOVA) in SPSS version 16. Tukey test was used to investigate the significance of inter-group differences. The level of statistical significance $(P)$ was considered $<0.05$. Data were expressed as mean \pm standard error of measurements.

\section{Results}

The thiol levels of hippocampus, striatum, cortex, and cerebellum tissues were significantly lower in the PD group than the control group, and significantly higher in B. carduchrum extract-treated groups $(100,200,400 \mathrm{mg} /$ $\mathrm{kg}$ ) than the groups with PD (Figures 1-4).

\section{Discussion}

Consistent with previous studies, the present study showed that PD led to significant decrease in thiol levels in the tissues of the brain's regions. This study also showed that B. carduchrum treatment compensated for this decrease through increasing thiol levels.

Oxidative stress is one of the factors that play a substantial role in degeneration of dopaminergic neurons in PD. It has been demonstrated that the damage due to oxidative stress and free radicals contributes greatly to the degeneration of the neurons of the substantia nigra, because the dopamine metabolism in the neurons is associated with the production of reactive oxygen species including hydrogen

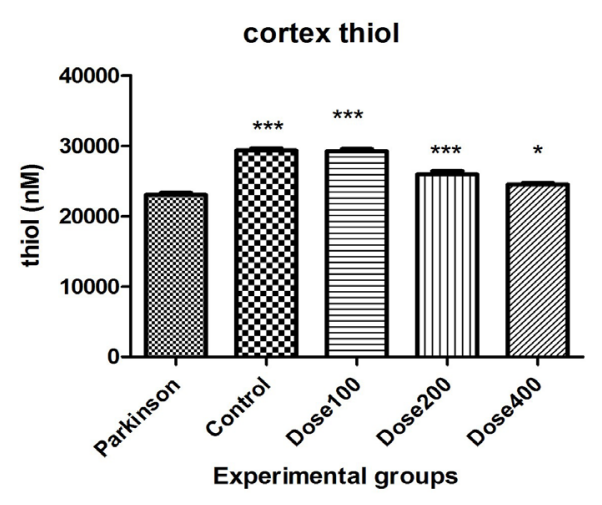

Figure 1. Comparison of mean ( \pm standard deviation) of thiol levels of cortex in control group, Parkinson's disease (PD) group, and Barium carduchrum extract $(100,200$, and $400 \mathrm{mg} / \mathrm{kg})$-treated PD groups. * Significant difference between PD group and the other groups. ${ }^{*} P<0.05$ and ${ }^{* * *} P<0.001$.

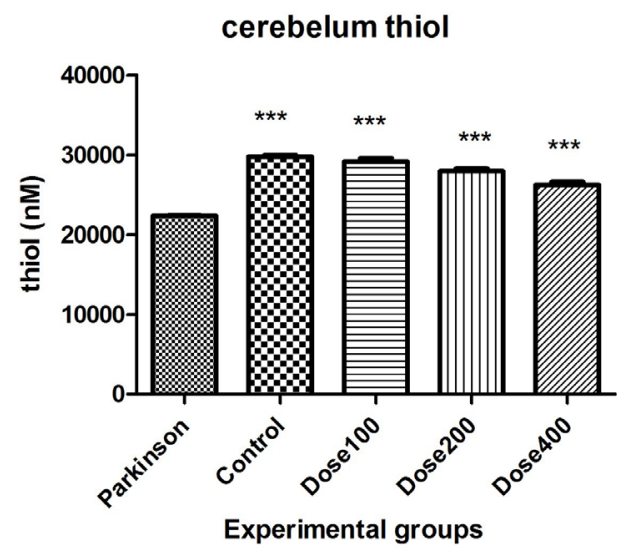

Figure 2. Comparison of mean ( \pm standard deviation) thiol levels of cerebellum in control group, Parkinson's disease (PD) group, and Barium carduchrum extract (100, 200, and $400 \mathrm{mg} / \mathrm{kg}$ )-treated PD groups. * Significant difference between PD group and the other groups; *** $P<0.001$.

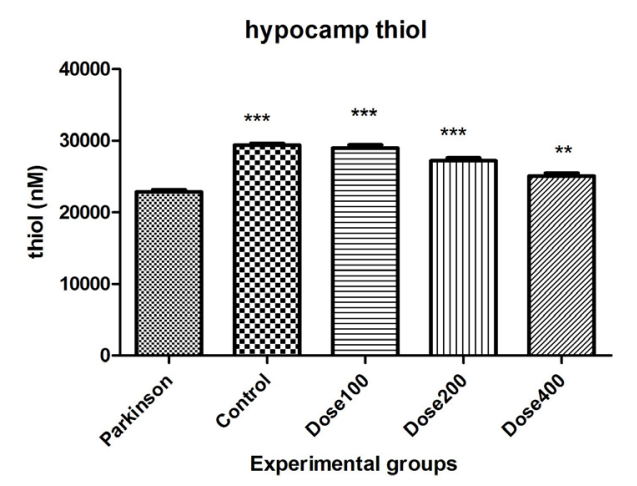

Figure 3. Comparison of mean ( \pm standard deviation) thiol levels of hippocampus in control group, Parkinson's disease (PD) group, and Barium carduchrum extract $(100,200$, and $400 \mathrm{mg} / \mathrm{kg})$-treated PD groups; * Significant difference between PD group and the other groups; ${ }^{*} P<0.05$ and ${ }^{* *} P<0.001$.

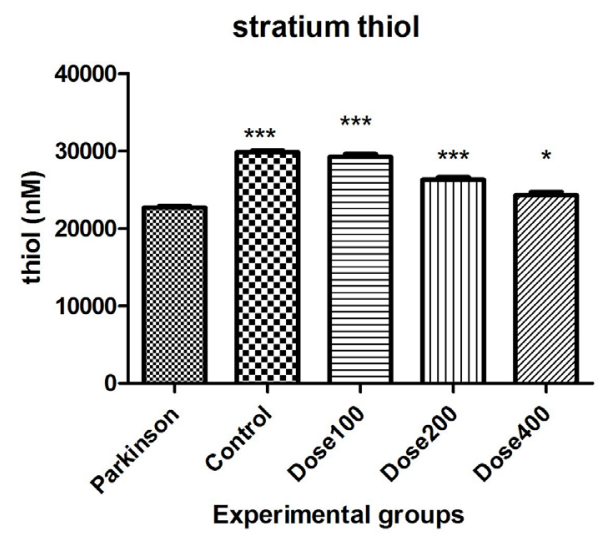

Figure 4. Comparison of mean ( \pm standard deviation) thiol levels of stratium in control group, Parkinson's disease (PD) group, and Barium carduchrum extract (100, 200, and $400 \mathrm{mg} / \mathrm{kg}$ )-treated PD groups; * Significant difference between PD group and the other groups; ${ }^{*} P<0.05$ and ${ }^{* * *} P<0.001$. 
peroxide and hydroxyl radical. Therefore, dopaminergic neurons in the brain are more predisposed to oxidative stress-associated cell death (4).

Oxidative damage to lipids, proteins, and DNA has been detected in the autopsies of PD patients. Interestingly, decreased levels of GSH, which is an antioxidant, is one of the first detected biochemical changes due to PD. Since then, a number of studies have investigated the role of GSH homeostasis in the progression of PD. GSH is the main antioxidant defense and the most frequent nonprotein thiol in mammalian cells including a free thiol group that is responsible for its regeneration (11).

Thiols are organosulfur compounds that include a carbonbonded SH group and are currently being considered a constituent of functional groups of cysteine amino acid group (cysteines protein, thiols protein) that are used in response to oxidative stress. Decrease in GSH levels is one of the first detected biochemical changes due to PD (11). Neurotoxin-6-hydroxydopamine degenerates the nigrostriatum through free radicals. This neurotoxin enters the dopaminergic terminals in the neostriatum through selective dopamine carriers, and leads to lipid peroxidation, DNA fragmentation, protein oxidation, and ultimately cell death through producing hydroxyl and hydrogen peroxide (12).

Hosseini et al determined the antioxidant activity of $B$. carduchrum extract using DPPH free radical inhibition and metal ion regeneration and chelation, and compared the antioxidant activities of $B$. carduchrum extract with those of ascorbic acid, alpha-tocopherol, EDTA, and BHT. The study of Hosseini et al showed that this extract was better at inhibiting $\mathrm{DPPH}$, and regenerating and chelating metal ions than BHT and alpha-tocopherol (13). The flavonoids of $B$. carduchrum extract react with hydroxyl anion, phenoxyl radical, and hypochlorous acid and prevent mitochondrial free radical-induced lipid peroxidation (13).

Studies have shown that inhibition of monoamine oxidase (MAO) by compounds such as alkaloids, phenolic acids and flavonoids may have therapeutic effects on PD (14-16). $\mathrm{MAO}_{\mathrm{A}}$ and $\mathrm{MAO}_{\mathrm{B}}$ are the central and peripheral nervous system enzymes that catalyze conversion of neurotransmitters to $\mathrm{H}_{2} \mathrm{O}_{2}$ or hydrogen peroxide aldehydes. In the brain of people with $\mathrm{PD}, \mathrm{MAO}$ inhibitors inhibit the breakdown of dopamine into its metabolites (17), and therefore can be used to maintain optimum levels of dopamine levels in clinical settings (18). As it has already been mentioned, B. carduchrum contains alkaloids, flavonoids and polyphenols, therefore the protective effect of this plant on PD may be due to the presence of these compounds and MAO inhibitory effect. In the present study, for the first time, the protective effect of $B$. carduchrum on neurodegenerative diseases was demonstrated, which may be due to the antioxidant effects of the plant. The protective effects of this plant may also be related to other mechanisms that should be considered in future studies. In this regard there are a lot of other plants with antioxidant activity (19-21). These plants or their components might have the same effects which should be examined.

\section{Conclusion}

It seems that B. carduchrum extract leads to functional regeneration of the nigrostriatal pathway through the mechanisms associated with its antioxidant properties.

\section{Acknowledgments}

The authors would like to thank the staff of laboratory of Islamic Azad University Izeh, Iran for their help.

\section{Authors' contributions}

MS: Statistical analysis, data interpretation, manuscript preparation. ZZ: Data collection, design of the study, literature search, funding collection. The authors read and confirmed publication of the manuscript.

\section{Ethical considerations}

All experimental procedures were conducted with the approval of the Ethics Committee of Azad University of Izeh with an ethical code of 57524 .

\section{Funding/Support}

This research was financially supported by Azad University of Izeh, Iran.

\section{References}

1. Dauer W, Przedborski S. Parkinson's disease: mechanisms and models. Neuron. 2003;39(6):889-909.

2. Datla KP, Zbarsky V, Rai D, Parkar S, Osakabe N, Aruoma OI, et al. Short-term supplementation with plant extracts rich in flavonoids protect nigrostriatal dopaminergic neurons in a rat model of Parkinson's disease. J Am Coll Nutr. 2007;26(4):341-9.

3. Valente EM, Abou-Sleiman PM, Caputo V, Muqit MM, Harvey K, Gispert S, et al. Hereditary early-onset Parkinson's disease caused by mutations in PINK1. Science. 2004;304(5674):1158-60. doi: 10.1126/science.1096284.

4. Dias V, Junn E, Mouradian MM. The role of oxidative stress in Parkinson's disease. J Parkinsons Dis. 2013;3(4):461-91. doi: 10.3233/jpd-130230.

5. Li Q, Zhao D, Bezard E. Traditional Chinese medicine for Parkinson's disease: a review of Chinese literature. Behav Pharmacol. 2006;17(5-6):403-10.

6. Boyce PC. A taxonomic revision of Biarum. Curtis's Botanical Magazine. 2008;25(1):2-17. doi: 10.1111/j.14678748.2007.00607.x.

7. Williams CA, Harborne JB, Mayo SJ. Anthocyanin pigments and leaf flavonoids in the family araceae. Phytochemistry. 1981;20(2):217-34. doi: 10.1016/0031-9422(81)85096-0.

8. Halvorsen BL, Carlsen MH, Phillips KM, Bohn SK, Holte K, Jacobs DR Jr, et al. Content of redox-active compounds (ie, antioxidants) in foods consumed in the United States. Am J Clin Nutr. 2006;84(1):95-135. doi: 10.1093/ajcn/84.1.95. 
9. Seifi Zangeneh M, Rafieirad M, Sazgar H. The effect of Kardeh (Biarum bovei) Hydro-alcoholic extract on pain threshold in STZ induced diabetic rats. J Herb Drugs. 2015;6(3):137-42.

10. Bilang-Bleuel A, Revah F, Colin P, Locquet I, Robert JJ, Mallet $\mathrm{J}$, et al. Intrastriatal injection of an adenoviral vector expressing glial-cell-line-derived neurotrophic factor prevents dopaminergic neuron degeneration and behavioral impairment in a rat model of Parkinson disease. Proc Natl Acad Sci U S A. 1997;94(16):8818-23.

11. Offen D, Ziv I, Sternin H, Melamed E, Hochman A. Prevention of dopamine-induced cell death by thiol antioxidants: possible implications for treatment of Parkinson's disease. Exp Neurol. 1996;141(1):32-9. doi: 10.1006/exnr.1996.0136.

12. Hald A, Lotharius J. Oxidative stress and inflammation in Parkinson's disease: is there a causal link? Exp Neurol. 2005;193(2):279-90. doi: 10.1016/j.expneurol.2005.01.013.

13. Hosseini E, Rousta E, Tabib Loghmany F, Mahmoudpour M. In Vitro Antioxidant Activity of Hydromethanolic Extract of Karde (Biarum carduchrum) and Tts Effects on the Serum Lipids of Rats. Iranian Journal of Nutrition Sciences \& Food Technology. 2014;9(3):1-8.

14. Bartikova H, Skalova L, Valentova K, Matouskova P, Szotakova B, Martin J, et al. Effect of oral administration of green tea extract in various dosage schemes on oxidative stress status of mice in vivo. Acta Pharm. 2015;65(1):65-73. doi: 10.1515/acph-2015-0007.

15. Kim GH, Kim JE, Rhie SJ, Yoon S. The Role of Oxidative Stress in Neurodegenerative Diseases. Exp Neurobiol. 2015;24(4):325-40. doi: 10.5607/en.2015.24.4.325.

16. Lee SJ, Chung HY, Lee IK, Oh SU, Yoo ID. Phenolics with Inhibitory Activity on Mouse Brain Monoamine Oxidase (MAO) from Whole Parts of Artemisia vulgaris $\mathrm{L}$ (Mugwort). Food Sci Biotechnol. 2000;9(3):179-82.

17. Cohen G, Farooqui R, Kesler N. Parkinson disease: a new link between monoamine oxidase and mitochondrial electron flow. Proc Natl Acad Sci U S A. 1997;94(10):48904.

18. Cohen G. Monoamine oxidase, hydrogen peroxide, and Parkinson's disease. Adv Neurol. 1987;45:119-25.

19. Bahmani M, Rafieian-Kopaei M, Jeloudari M, Eftekhari Z, Delfan B, Zargaran A, et al. A review of the health effects and uses of drugs of plant licorice (Glycyrrhiza glabra L.) in Iran. Asian Pac J Trop Dis 2014;4(Suppl 2):847-9.

20. Saki K, Bahmani M, Rafieian-Kopaei M, Hassanzadazar H, Dehghan K, Bahmani F, et al. The most common native medicinal plants used for psychiatric and neurological disorders in Urmia city, northwest of Iran. Asian Pac J Trop Dis 2014;4(Suppl 2):895-901.

21. Bahmani M, Saki K, Rafieian-Kopaei M, Karamati SA, Eftekhari Z, Jelodari M. The most common herbal medicines affecting Sarcomastigophora branches: a review study. Asian Pac J Trop Med 2014;7(Suppl 1):14-21. 